\title{
Prediction of disability-adjusted life years for diseases due to low fruit intake in 2017-2040 in Japan
}

\author{
Daisuke Yoneoka ${ }^{1,2, \dagger}$, (․, Shuhei Nomura $2,3,4, *, \dagger$, Shiori Tanaka ${ }^{2,4}$, Aya Ishizuka ${ }^{2}$ \\ Ueda Peter ${ }^{2,5}$, Santosh Kumar Rauniyar ${ }^{2}$, Keiji Nakamura ${ }^{6,7}$, Hisayuki Uneyama ${ }^{7}$, \\ Naoki Hayashi ${ }^{7,8}$ and Kenji Shibuya ${ }^{2,9}$
}

'Graduate School of Public Health, St. Luke's International University, Tokyo, Japan: ${ }^{2}$ Department of Global Health Policy, Graduate School of Medicine, The University of Tokyo, Tokyo, Japan: ${ }^{3}$ Department of Health Policy and Management, School of Medicine, Keio University, 35 Shinanomachi, Shinjuku-ku, Tokyo 160-8582, Japan: ${ }^{4}$ Epidemiology and Prevention Group, Center for Public Health Sciences, National Cancer Center, Tokyo, Japan: ${ }^{5}$ Department of Medicine, Clinical Epidemiology Division, Karolinska Institutet, Stockholm, Sweden: ${ }^{6} \mathrm{Graduate}$ School of Environmental and Information Studies, Tokyo City University, Yokohama, Japan: ${ }^{7}$ Ajinomoto Co., Inc., Tokyo, Japan: ${ }^{8}$ Department of Applied Biological Chemistry, Graduate School of Agriculture and Life Sciences, The University of Tokyo, Tokyo, Japan: 'Department of Global Health Policy, Institute for Population Health, King's College London, London, UK

Submitted 17 February 2020: Final revision received 30 0ctober 2020: Accepted 5 November 2020: First published online 13 November 2020

\begin{abstract}
Objective: The current study aimed to predict disability-adjusted life years (DALY) rate in Japan through 2040 with plausible future scenarios of fruit intake for neoplasms, cardiovascular diseases (CVD) and diabetes and kidney diseases (DKD). Design: Data from National Health and Nutrition Surveys and the Global Burden of Diseases study in 2017 were used. We developed an autoregressive integrated moving average model with four future scenarios. Reference scenario maintains the current trend. Best scenario assumes that the goal defined in Health Japan 21 is achieved in 2023 and is kept constant afterwards. Moderate scenario assumes that the goal is achieved in 2040. Constant scenario applies the same proportion of 2016 for the period between 2017 and 2040.

Setting: DALY rates in Japan were predicted for the period between 2017 and 2040. Participants: Population aged more than than 20 years old.

Results: In our reference forecast, the DALY rates in all-ages group were projected to be stable for CVD and continue increasing for neoplasms and DKD. Age groupspecific DALY rates for these three disease groups were forecasted to decrease, with some exceptions. Among men aged 20-49 years, DALY attributable to CVD differed substantially between the scenarios, implying that there is a significant potential for reducing the burden of CVD by increasing fruit intake at the population level.

Conclusions: Our scenario analysis shows that higher fruit intake is associated with lower disease burden in Japan. Further research is required to assess which policies and interventions can be used to achieve an increase in fruit intake as modelled in the scenarios of the current study.
\end{abstract}

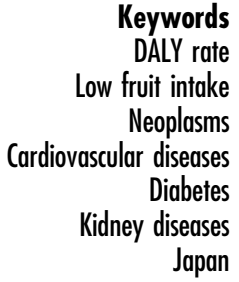

occupational factors, on disease burden measured as disability-adjusted life years (DALY). One DALY can be considered as one lost year of 'healthy' life. The study found that low fruit intake was the ninth most important risk factor $^{(1,2)}$ accounting for $2 \cdot 3 \%$ of the total disease burden ${ }^{(1)}$. In the current study, we take the same definition for fruits as the Global Burden of Disease study, where 'fruit' means

Daisuke Yoneoka and Shuhei Nomura shared first authorship. 
fresh, frozen, cooked, canned or dried fruits, excluding fruit juices and salted or pickled fruits.

People in Japan eat less fruits as compared with other national populations ${ }^{(3)}$. According to the Food and Agriculture Organisation (FAO) of the UN, in 2013, the average fruit supply per capita in Japan was $144.8 \mathrm{~g} / \mathrm{d}$, corresponding to a 135th place among 175 countries. Accordingly, the Japanese fruit supply per person was approximately half of the amount in the USA ${ }^{(3)}$. Japan's National Health and Nutrition Survey showed that the average Japanese fruit intake per capita peaked at $193.5 \mathrm{~g} / \mathrm{d}$ in $1975^{(4)}$. Since then, the intake has been declining and, for the first time in the past 40 years, has dropped below $100 \mathrm{~g}$ to reach $98.9 \mathrm{~g}$ in $2016^{(5)}$. In Japan, fruit intake increased with age, and women consumed more fruit than men in all 10-year-old age groups in $2015^{(6)}$. The national health promotion policy guideline 'Health Japan 21 (second phase)', which was established in 2012 to improve lifestyle and extend healthy life expectancy, has set a target of having $<30 \%$ of the population consuming $<100 \mathrm{~g}$ of fruit $/ \mathrm{d}^{(7)}$. However, even among those who consumed the most fruit, identified as those in their 70 s, only $32 \%$ met the recommended level ${ }^{(6)}$. This target, defined on the population level, is not comparable with the individual-level goal defined in the World Health Organisation (WHO)'s guideline that recommends eating at least $400 \mathrm{~g}$ of fruit and vegetables per day (excluding potatoes and other starchy tubers $^{(8)}$.

Based on four different scenarios for future fruit consumption in Japan, the current study aims to predict the DALY through 2040 (when many Japanese health policies, such as 'Social Security and Workplace Reform with a View to 2040 '(9) and 'Healthy Life Extension Plan'(10), often set their goal to be achieved) for chronic diseases that are well known to be associated with low fruit intake ${ }^{(1)}$. Since low fruit intake is a modifiable risk factor, medical and political interventions to modify this risk factor could have a large potential to prevent disease, prolong healthy life and efficiently maintain and improve population health ${ }^{(11)}$. By providing estimates of the effects of altered fruit consumption, the current study aims to provide data that could guide decisions on policy design and prioritisation.

\section{Methods}

\section{Overview}

According to the Global Burden of Disease study, the three disease groups, or three leading causes of health lost, that have been identified to be associated with low fruit intake were neoplasms, cardiovascular diseases and diabetes and kidney diseases from level 2 in the Global Burden of Disease hierarchical causal structure. The data for these diseases from 1990 to 2016 were used to predict the future values of DALY rates for the period from 2017 to 2040.
Following the Global Burden of Disease's prediction methodology ${ }^{(12)}$, we developed a three-component model of cause-specific DALY for these three causes. This model included a component explained by changes in major behavioural and metabolic risk predictors including the proportion of those who consume less than $100 \mathrm{~g}$ of fruit; a component explained by income per person, educational attainment and total fertility rate under 25 years, which were aggregated into the socio-demographic index that ranges from 0 to 1 and an autoregressive integrated moving average model to estimate the unexplained component over the time span. Further details, including data sources and model formulae, are explained below.

\section{Data sources}

\section{Disability-adjusted life years and socio-demographic} index data, 1990-2016

The estimates of the DALY rate (per 100000 population) for neoplasms, cardiovascular diseases and diabetes and kidney diseases as well as the socio-demographic index in Japan for the years of 1990-2016 were extracted from the Global Burden of Disease 2017 study $^{(13)}$. Detailed information regarding the estimation of DALY and sociodemographic index have been presented elsewhere ${ }^{(13,14)}$. Data extraction and analysis were performed by sex (male, female and sex combined) and age group (20-49 years old, 50-69 years old, $\geq 70$ years old and all ages). We followed the age categorisation scheme used in the Global Burden of Disease study to guarantee comparability. Our study did not use data for the 0-19-year-old age group because of the lack of risk predictor data (see below).

\section{Behavioural and metabolic risk predictor, 1990-2016}

We extracted the following predictors from Japan's National Health and Nutrition Survey for 1990-2016: the proportion of those who consume $<100 \mathrm{~g}$ (per day) of fruit and the prevalence of current smokers, current alcohol drinkers (consumption of $180.39 \mathrm{ml}$ or more of alcoholic beverages for three or more days a week) and obesity for each sex- and age-group. In the current study, obesity was defined as BMI of $\geq 25 \mathrm{~kg} / \mathrm{m}^{2}$ based on the Japanese Society for the Study of Obesity ${ }^{(15,16)}$. The National Health and Nutrition Survey is an annual and national representative household survey conducted by the Japanese Ministry of Health, Labour and Welfare to clarify dietary habits, nutrition intake and lifestyle ${ }^{(17)}$. The intake of nutrients, foods and alcohol was estimated based on the dietary record and the corresponding food composition list in the Standard Tables of Food Composition in Japan (sixth revised edition as at 2016) ${ }^{(18)}$. Since the lifestyle questionnaire, which was used to estimate the prevalence of smoking and alcohol consumption, was not conducted for the population aged less than 20 years old, only those aged $\geq 20$ years old were considered in the current study. 


\section{Statistical analysis and scenarios}

Autoregressive integrated moving average model for the prediction of 2017-2040

The autoregressive integrated moving average model was used to predict the future values of the DALY rates and the risk predictors discussed above. The autoregressive integrated moving average is a statistical model that leverages time series data to forecast future trends by incorporating the past information. The past information is explicitly incorporated into the model with time lags, which allows for flexible modelling strategy to predict the future values. The autoregressive integrated moving average model combines a moving average model, which controls the dependent relationship between an observation and some lagged observations, with an autoregression model, which controls the dependency between an observation and a residual error, to model the temporal dependence over the time span using the shift and lag of historical information.

The autoregressive integrated moving average model requires the specification three parameters $p, d$ and $q$, which is denoted as ARIMA $(p, d, q)$ and generally given by

$$
\left(1-\sum_{i=1}^{p} \alpha_{i} L^{i}\right)(1-L)^{d} y_{t}=\left(1+\sum_{i=1}^{q} \beta_{i} L^{d}\right) \varepsilon_{t}
$$

where $y_{t}$ is the outcome of interest (i.e. each risk predictor), $\varepsilon_{t}$ is an (white noise) error term with constant intensity of $\sigma^{2}$ at time $t, L$ is a time lag operator defined as $L^{k} y_{t}=y_{t-k}$ and $\alpha_{i}$ s and $\beta_{i}$ s are the coefficient parameters $^{(19)}$. Before fitting each model, the stationarity of the time series was tested by Dickey-Fuller test ${ }^{(19)}$. If nonstationary was plausible, we transformed the data to satisfy the stationarity by taking a suitable difference between time points with order $d$. The autocorrelation function and partial autocorrelation function were used to estimate the stationary status and to decide the grid search range for the parameters in the models. The model parameters were estimated by maximum likelihood method. Akaike's Information Criterion was calculated to select the best model with the parameters $p, d$ and $q$.

We applied a two-step approach to predict the future DALY rates. The first step was to independently predict the future values of each predictor from 2017 to 2040 using equation (1), and then the second step was to predict the log-scaled DALY rate by using the following equation (2) after plugging the predicted values of the above predictors into $x_{t j}$ :

$$
\begin{aligned}
\left(1-\sum_{i=1}^{p} \alpha_{i} L^{i}\right)(1-L)^{d} y_{t}= & \sum_{j=1}^{4} \gamma_{j} L^{d} x_{t j} \\
& +\left(1+\sum_{i=1}^{q} \beta_{i} L^{d}\right) \varepsilon_{t}
\end{aligned}
$$

where $y_{t}$ is the DALY rate at time $t, x_{t j}$ is the value of $j$ th risk predictor at time $t$ (i.e. they are predicted in the first step) and $\gamma_{j}$ is a coefficient for the $j$ th risk predictor. All analyses were conducted by $\mathrm{R}$ (version 3.6.1). Using the maximum likelihood method, the parameters in equations (1) and (2) were separately estimated by disease, age and sex categories.

\section{Future scenarios for the proportion of those who consume $<100 \mathrm{~g}$ of fruit/d}

We assumed four future scenarios to evaluate the impact of change in the proportion of those who consume $<100 \mathrm{~g}$ of fruit/d on the DALY rates for the three diseases (neoplasms, cardiovascular diseases and diabetes and kidney diseases) from 2017 to 2040 in Japan: one reference and three alternative scenarios (best, moderate and constant scenarios). The reference prediction assumed that the current trend is maintained. The future proportion was predicted by the autoregressive integrated moving average model defined in equation (1). The best scenario assumed that the goal defined in Health Japan 21 (i.e. the proportion of those who consume $<100 \mathrm{~g}$ of fruit/d is $<30 \%$ ) is achieved in 2023 and the proportion is kept constant afterwards $^{(7)}$. The moderate scenario assumed the goal defined in Health Japan 21 is achieved in 2040 instead of 2023, assuming a monotonic decrease function from 2017 to 2040. The constant scenario is a scenario in which the latest proportion (i.e. the value at 2016) is applied constantly from 2017 to 2040.

By plugging these assumed scenario values into equation (2) as predictors with other risk predictors, the final prediction values of DALY rate for these scenarios through 2040 can be obtained. It should be noted that the proportion consuming $<100 \mathrm{~g}$ of fruit/d in 2040 is the same for the best and the moderate scenarios (i.e. both scenarios assumed that the proportions are $<30 \%$ at 2040), and the projected DALY rates converge mathematically to the same values in 2040 while the prediction trajectories until 2039 are different.

\section{Results}

Table 1 shows the socio-demographic index and behavioural and metabolic risk predictors by sex- and age-groups from 1990 to 2016. While the prevalence of current smokers and alcohol drinkers has declined since 1990, the sociodemographic index and the prevalence of obesity have increased.

Table 2 shows both the observed (1990-2016) and predicted (2017-2014) fruit intake for the four scenarios. Overall, women and older age groups consumed more fruits than men and younger age groups. Note that although the proportions of those consuming $<100 \mathrm{~g}$ of fruits/d were at similar levels for all-ages groups in 1990, the subsequent trajectories differed by age groups from 1990 to 2016. In 


\section{Public Health Nutrition}

Table 1 Sex- and age group-specific disability-adjusted life years (DALY) rate, socio-demographic index, behavioural and metabolic risk predictor data*

\begin{tabular}{|c|c|c|c|c|c|c|c|c|c|c|c|}
\hline \multirow[b]{3}{*}{ Year } & \multirow[b]{3}{*}{ Sex } & \multicolumn{3}{|c|}{ GBD 2017 data } & \multirow[b]{3}{*}{$\begin{array}{l}\text { SDI } \\
(\%)\end{array}$} & \multirow{2}{*}{\multicolumn{6}{|c|}{ National Health and Nutrition Survey (NHNS) data }} \\
\hline & & \multicolumn{3}{|c|}{$\begin{array}{l}\text { All ages DALY rate per } \\
100,000 \text { population }^{(4)}\end{array}$} & & & & & & & \\
\hline & & Neoplasms & $\begin{array}{l}\text { Cardiovascular } \\
\text { diseases }\end{array}$ & $\begin{array}{l}\text { Diabetes and kidney } \\
\text { diseases }\end{array}$ & & $\begin{array}{c}\text { Number of NHNS } \\
\text { participant }\end{array}$ & $\begin{array}{l}\text { Mean } \\
\text { age }\end{array}$ & SD & $\begin{array}{l}\text { Obesity } \\
(\%)\end{array}$ & $\begin{array}{c}\text { Current smoker } \\
(\%)\end{array}$ & $\begin{array}{l}\text { Current alcohol } \\
\text { drinker (\%) }\end{array}$ \\
\hline \multirow[t]{3}{*}{1990} & Male & $5130 \cdot 206$ & $4649 \cdot 616$ & $950 \cdot 842$ & $80 \cdot 3$ & 6182 & $47 \cdot 4$ & $16 \cdot 1$ & $22 \cdot 3$ & $53 \cdot 1$ & $52 \cdot 1$ \\
\hline & Female & $3280 \cdot 867$ & 3674.784 & 828.055 & $80 \cdot 3$ & 7025 & 48.7 & $16 \cdot 8$ & $21 \cdot 8$ & $9 \cdot 7$ & $6 \cdot 1$ \\
\hline & Both sexes combined & $4190 \cdot 121$ & 4154.074 & 888.425 & $80 \cdot 3$ & 13207 & $48 \cdot 1$ & 16.5 & $22 \cdot 0$ & 28.5 & $26 \cdot 0$ \\
\hline \multirow{3}{*}{1995} & Male & 5831.968 & 4625.992 & 1014.659 & $82 \cdot 3$ & 4976 & $47 \cdot 8$ & 16.5 & 23.9 & 52.7 & 54.4 \\
\hline & Female & $3578 \cdot 775$ & 3573.717 & 839.433 & $82 \cdot 3$ & 5766 & 49.0 & $17 \cdot 3$ & 20.9 & 10.7 & 7.4 \\
\hline & Both sexes combined & $4685 \cdot 216$ & $4090 \cdot 442$ & 925.478 & $82 \cdot 3$ & 10742 & 48.4 & $17 \cdot 0$ & $22 \cdot 2$ & 28.2 & $27 \cdot 0$ \\
\hline \multirow{3}{*}{2000} & Male & $6185 \cdot 241$ & 4523.508 & 1058.045 & 83.4 & 4513 & $50 \cdot 1$ & $17 \cdot 0$ & $26 \cdot 8$ & 47.4 & $50 \cdot 8$ \\
\hline & Female & $3745 \cdot 152$ & 3344.292 & 844.288 & 83.4 & 5149 & $51 \cdot 7$ & 17.5 & $21 \cdot 3$ & 11.5 & 9.0 \\
\hline & Both sexes combined & $4940 \cdot 908$ & $3922 \cdot 162$ & 949.039 & 83.4 & 9662 & $50 \cdot 8$ & $17 \cdot 2$ & 23.8 & $27 \cdot 0$ & $27 \cdot 0$ \\
\hline \multirow{3}{*}{2005} & Male & $6454 \cdot 112$ & $4646 \cdot 185$ & 1097.638 & 84.4 & 3591 & 52.5 & $17 . \overline{5}$ & $28 \cdot 6$ & 39.3 & $36 \cdot 7$ \\
\hline & Female & $3866 \cdot 874$ & 3313.558 & 841.733 & 84.4 & 4155 & 54.5 & $17 \cdot 8$ & $22 \cdot 0$ & 11.4 & 7.4 \\
\hline & Both sexes combined & 5131.424 & 3964.899 & $966 \cdot 811$ & 84.4 & 7746 & 53.6 & $17 \cdot 7$ & 24.9 & $24 \cdot 3$ & $20 \cdot 9$ \\
\hline \multirow{3}{*}{2010} & Male & $6549 \cdot 874$ & 4664.061 & 1228.505 & $85 \cdot 3$ & 3740 & 53.9 & 17.5 & 30.4 & $32 \cdot 2$ & 35.4 \\
\hline & Female & 3954.495 & $3300 \cdot 696$ & 934.343 & $85 \cdot 3$ & 4239 & $55 \cdot 6$ & $17 \cdot 8$ & $21 \cdot 1$ & 8.4 & $7 \cdot 0$ \\
\hline & Both sexes combined & $5220 \cdot 232$ & 3965.593 & $1077 \cdot 802$ & $85 \cdot 3$ & 7746 & 54.8 & $17 \cdot 7$ & $25 \cdot 3$ & 19.5 & $20 \cdot 3$ \\
\hline \multirow[t]{3}{*}{2016} & Male & 6449.688 & $4590 \cdot 286$ & 1235.046 & $86 \cdot 3$ & 12132 & $56 \cdot 6$ & $17 \cdot 6$ & 31.7 & 30.5 & 34.0 \\
\hline & Female & $3940 \cdot 317$ & $3400 \cdot 755$ & $1003 \cdot 632$ & $86 \cdot 3$ & 14010 & 58.1 & $18 \cdot 0$ & 21.3 & 7.6 & 7.5 \\
\hline & Both sexes combined & $5163 \cdot 140$ & $3980 \cdot 416$ & 1116.401 & $86 \cdot 3$ & 26142 & 57.4 & $17 \cdot 8$ & $25 \cdot 9$ & $18 \cdot 2$ & $19 \cdot 8$ \\
\hline
\end{tabular}

GBD, Global Burden of Disease study; NHNS, National Health and Nutrition Survey of Japan; SDI, socio-demographic index.

*Note that we used data for each year, but the table lists only selected years. 
Table 2 Observed (1990-2016) and predicted (2017-2014) proportion (\%) of those who consume $<100 \mathrm{~g}$ of fruit/d

\begin{tabular}{|c|c|c|c|c|c|c|c|c|c|c|c|c|c|c|c|c|c|c|c|c|c|c|c|c|c|}
\hline \multirow{3}{*}{$\begin{array}{l}\text { Age category } \\
\text { Sex } \\
\text { Scenario }\end{array}$} & & \multicolumn{12}{|c|}{ 20-49 years } & \multicolumn{12}{|c|}{$50-69$ years } \\
\hline & & \multicolumn{4}{|c|}{ Male } & \multicolumn{4}{|c|}{ Female } & \multicolumn{4}{|c|}{ Both sexes combined } & \multicolumn{4}{|c|}{ Male } & \multicolumn{4}{|c|}{ Female } & \multicolumn{4}{|c|}{ Both sexes combined } \\
\hline & & $\mathrm{R}$ & 1 & 2 & 3 & $\mathrm{R}$ & 1 & 2 & 3 & $\mathrm{R}$ & 1 & 2 & 3 & $\mathrm{R}$ & 1 & 2 & 3 & $\mathrm{R}$ & 1 & 2 & 3 & $\mathrm{R}$ & 1 & 2 & 3 \\
\hline \multirow{7}{*}{ Observed period } & 1990 & $44 \cdot 1$ & $44 \cdot 1$ & $44 \cdot 1$ & $44 \cdot 1$ & $49 \cdot 8$ & $49 \cdot 8$ & $49 \cdot 8$ & $49 \cdot 8$ & $47 \cdot 1$ & $47 \cdot 1$ & $47 \cdot 1$ & $47 \cdot 1$ & 39.6 & 39.6 & 39.6 & 39.6 & $42 \cdot 0$ & $42 \cdot 0$ & $42 \cdot 0$ & $42 \cdot 0$ & 40.9 & 40.9 & 40.9 & 40.9 \\
\hline & 1995 & 65.8 & $65 \cdot 8$ & $65 \cdot 8$ & 65.8 & $52 \cdot 6$ & 52.6 & 52.6 & 52.6 & 58.9 & 58.9 & 58.9 & 58.9 & $47 \cdot 3$ & $47 \cdot 3$ & $47 \cdot 3$ & $47 \cdot 3$ & 32.7 & $32 \cdot 7$ & $32 \cdot 7$ & 32.7 & 39.6 & 39.6 & 39.6 & 39.6 \\
\hline & 2000 & 77.8 & $77 \cdot 8$ & 77.8 & 77.8 & $65 \cdot 6$ & 65.6 & 65.6 & 65.6 & 71.4 & 71.4 & 71.4 & 71.4 & 50.9 & 50.9 & 50.9 & 50.9 & 34.9 & 34.9 & 34.9 & 34.9 & $42 \cdot 6$ & $42 \cdot 6$ & $42 \cdot 6$ & 42.6 \\
\hline & 2005 & 79.7 & 79.7 & 79.7 & 79.7 & $69 \cdot 2$ & $69 \cdot 2$ & 69.2 & 69.2 & 74.2 & 74.2 & 74.2 & 74.2 & 55.9 & 55.9 & 55.9 & 55.9 & 41.0 & 41.0 & $41 \cdot 0$ & 41.0 & $47 \cdot 8$ & $47 \cdot 8$ & $47 \cdot 8$ & $47 \cdot 8$ \\
\hline & 2010 & 82.4 & 82.4 & 82.4 & 82.4 & $76 \cdot 3$ & $76 \cdot 3$ & $76 \cdot 3$ & $76 \cdot 3$ & 79.2 & $79 \cdot 2$ & $79 \cdot 2$ & $79 \cdot 2$ & 60.3 & $60 \cdot 3$ & 60.3 & $60 \cdot 3$ & $48 \cdot 3$ & $48 \cdot 3$ & $48 \cdot 3$ & 48.3 & 53.9 & 53.9 & 53.9 & 53.9 \\
\hline & 2016 & $85 \cdot 1$ & $85 \cdot 1$ & $85 \cdot 1$ & $85 \cdot 1$ & 78.9 & 78.9 & 78.9 & 78.9 & $81 \cdot 8$ & $81 \cdot 8$ & $81 \cdot 8$ & $81 \cdot 8$ & $65 \cdot 1$ & $65 \cdot 1$ & 65.1 & $65 \cdot 1$ & $52 \cdot 0$ & $52 \cdot 0$ & $52 \cdot 0$ & $52 \cdot 0$ & 58.0 & 58.0 & 58.0 & 58.0 \\
\hline & 2023 & $97 \cdot 7$ & $30 \cdot 0$ & $70 \cdot 7$ & $85 \cdot 1$ & $85 \cdot 3$ & $30 \cdot 0$ & $66 \cdot 1$ & 78.9 & 91.5 & 30.0 & $68 \cdot 3$ & 81.8 & 71.7 & $30 \cdot 0$ & 55.9 & $65 \cdot 1$ & $50 \cdot 3$ & $30 \cdot 0$ & $46 \cdot 2$ & $52 \cdot 0$ & $60 \cdot 4$ & 30.0 & 50.7 & 58.0 \\
\hline \multirow{3}{*}{ Prediction period } & 2030 & $100 \cdot 0$ & 30.0 & 53.9 & 85.1 & $92 \cdot 1$ & 30.0 & 51.2 & 78.9 & $100 \cdot 0$ & 30.0 & 52.5 & 81.8 & 77.6 & 30.0 & $45 \cdot 2$ & 65.1 & $50 \cdot 3$ & 30.0 & 39.6 & 52.0 & 64.4 & 30.0 & $42 \cdot 2$ & 58.0 \\
\hline & 2035 & $100 \cdot 0$ & 30.0 & $42 \cdot 0$ & $85 \cdot 1$ & 96.9 & 30.0 & $40 \cdot 6$ & 78.9 & $100 \cdot 0$ & $30 \cdot 0$ & 41.3 & 81.8 & 82.5 & 30.0 & 37.6 & $65 \cdot 1$ & $50 \cdot 3$ & $30 \cdot 0$ & 34.8 & $52 \cdot 0$ & $67 \cdot 2$ & 30.0 & $36 \cdot 1$ & 58.0 \\
\hline & 2040 & $100 \cdot 0$ & $30 \cdot 0$ & $30 \cdot 0$ & $85 \cdot 1$ & $100 \cdot 0$ & $30 \cdot 0$ & $30 \cdot 0$ & 78.9 & $100 \cdot 0$ & $30 \cdot 0$ & $30 \cdot 0$ & 81.8 & $86 \cdot 5$ & $30 \cdot 0$ & $30 \cdot 0$ & $65 \cdot 1$ & $50 \cdot 3$ & $30 \cdot 0$ & $30 \cdot 0$ & $52 \cdot 0$ & $70 \cdot 0$ & $30 \cdot 0$ & $30 \cdot 0$ & $58 \cdot 0$ \\
\hline \multicolumn{2}{|l|}{ Age category } & \multicolumn{12}{|c|}{$\geq 70$ years } & \multicolumn{12}{|c|}{ All ages } \\
\hline \multicolumn{2}{|l|}{ Sex } & \multicolumn{4}{|c|}{ Male } & \multicolumn{4}{|c|}{ Female } & \multicolumn{4}{|c|}{ Both sexes combined } & \multicolumn{4}{|c|}{ Male } & \multicolumn{4}{|c|}{ Female } & \multicolumn{4}{|c|}{ Both sexes combined } \\
\hline Scenario & & $\mathrm{R}$ & 1 & 2 & 3 & $\mathrm{R}$ & 1 & 2 & 3 & $\mathrm{R}$ & 1 & 2 & 3 & $\mathrm{R}$ & 1 & 2 & 3 & $\mathrm{R}$ & 1 & 2 & 3 & $\mathrm{R}$ & 1 & 2 & 3 \\
\hline \multirow{6}{*}{ Observed period } & 1990 & 41.7 & $41 \cdot 7$ & 41.7 & 41.7 & $51 \cdot 1$ & $51 \cdot 1$ & $51 \cdot 1$ & $51 \cdot 1$ & $47 \cdot 3$ & $47 \cdot 3$ & $47 \cdot 3$ & $47 \cdot 3$ & $42 \cdot 4$ & $42 \cdot 4$ & $42 \cdot 4$ & $42 \cdot 4$ & 47.4 & $47 \cdot 4$ & 47.4 & $47 \cdot 4$ & $45 \cdot 0$ & $45 \cdot 0$ & $45 \cdot 0$ & $45 \cdot 0$ \\
\hline & 1995 & $43 \cdot 8$ & $43 \cdot 8$ & $43 \cdot 8$ & $43 \cdot 8$ & $42 \cdot 4$ & $42 \cdot 4$ & $42 \cdot 4$ & $42 \cdot 4$ & 43.0 & 43.0 & $43 \cdot 0$ & $43 \cdot 0$ & $57 \cdot 3$ & $57 \cdot 3$ & $57 \cdot 3$ & $57 \cdot 3$ & 44.7 & 44.7 & $44 \cdot 7$ & 44.7 & 50.5 & 50.5 & 50.5 & 50.5 \\
\hline & 2000 & $45 \cdot 6$ & $45 \cdot 6$ & $45 \cdot 6$ & $45 \cdot 6$ & 41.4 & 41.4 & 41.4 & 41.4 & $43 \cdot 1$ & $43 \cdot 1$ & $43 \cdot 1$ & $43 \cdot 1$ & $62 \cdot 9$ & $62 \cdot 9$ & $62 \cdot 9$ & 62.9 & $50 \cdot 0$ & $50 \cdot 0$ & $50 \cdot 0$ & $50 \cdot 0$ & $56 \cdot 0$ & $56 \cdot 0$ & $56 \cdot 0$ & $56 \cdot 0$ \\
\hline & 2005 & 41.5 & 41.5 & 41.5 & 41.5 & 38.2 & 38.2 & 38.2 & 38.2 & 39.6 & $39 \cdot 6$ & 39.6 & 39.6 & $63 \cdot 0$ & 63.0 & 63.0 & 63.0 & $51 \cdot 3$ & $51 \cdot 3$ & $51 \cdot 3$ & $51 \cdot 3$ & $56 \cdot 7$ & $56 \cdot 7$ & $56 \cdot 7$ & $56 \cdot 7$ \\
\hline & 2010 & $46 \cdot 2$ & $46 \cdot 2$ & $46 \cdot 2$ & $46 \cdot 2$ & $42 \cdot 6$ & $42 \cdot 6$ & $42 \cdot 6$ & $42 \cdot 6$ & 44.2 & $44 \cdot 2$ & 44.2 & $44 \cdot 2$ & $66 \cdot 0$ & 66.0 & $66 \cdot 0$ & $66 \cdot 0$ & $57 \cdot 3$ & $57 \cdot 3$ & $57 \cdot 3$ & $57 \cdot 3$ & 61.3 & $61 \cdot 3$ & $61 \cdot 3$ & 61.3 \\
\hline & 2016 & $46 \cdot 3$ & $46 \cdot 3$ & $46 \cdot 3$ & $46 \cdot 3$ & $43 \cdot 8$ & 43.8 & 43.8 & $43 \cdot 8$ & 44.9 & $44 \cdot 9$ & 44.9 & 44.9 & $67 \cdot 0$ & $67 \cdot 0$ & $67 \cdot 0$ & $67 \cdot 0$ & 58.4 & $58 \cdot 4$ & 58.4 & $58 \cdot 4$ & $62 \cdot 3$ & $62 \cdot 3$ & $62 \cdot 3$ & $62 \cdot 3$ \\
\hline \multirow[t]{4}{*}{ Prediction period } & 2020 & $45 \cdot 3$ & $30 \cdot 0$ & $42 \cdot 1$ & $46 \cdot 3$ & $42 \cdot 4$ & $30 \cdot 0$ & $40 \cdot 2$ & $43 \cdot 8$ & $42 \cdot 8$ & $30 \cdot 0$ & $41 \cdot 0$ & 44.9 & $67 \cdot 0$ & $30 \cdot 0$ & $57 \cdot 4$ & $67 \cdot 0$ & $56 \cdot 6$ & $30 \cdot 0$ & $51 \cdot 0$ & $58 \cdot 4$ & $66 \cdot 3$ & $30 \cdot 0$ & $53 \cdot 9$ & $62 \cdot 3$ \\
\hline & 2023 & $45 \cdot 3$ & $30 \cdot 0$ & $37 \cdot 1$ & $46 \cdot 3$ & $42 \cdot 4$ & $30 \cdot 0$ & $36 \cdot 0$ & $43 \cdot 8$ & $42 \cdot 8$ & $30 \cdot 0$ & 36.5 & 44.9 & $67 \cdot 0$ & $30 \cdot 0$ & $46 \cdot 1$ & $67 \cdot 0$ & $56 \cdot 6$ & $30 \cdot 0$ & $42 \cdot 3$ & $58 \cdot 4$ & $70 \cdot 2$ & $30 \cdot 0$ & $44 \cdot 1$ & $62 \cdot 3$ \\
\hline & 2035 & $45 \cdot 3$ & $30 \cdot 0$ & 33.5 & $46 \cdot 3$ & $42 \cdot 4$ & $30 \cdot 0$ & 33.0 & $43 \cdot 8$ & $42 \cdot 8$ & $30 \cdot 0$ & $33 \cdot 2$ & 44.9 & $67 \cdot 0$ & $30 \cdot 0$ & 38.0 & $67 \cdot 0$ & $56 \cdot 6$ & $30 \cdot 0$ & $36 \cdot 2$ & 58.4 & 73.0 & $30 \cdot 0$ & $37 \cdot 0$ & $62 \cdot 3$ \\
\hline & 2040 & $45 \cdot 3$ & 30.0 & 30.0 & $46 \cdot 3$ & 42.4 & $30 \cdot 0$ & 30.0 & 43.8 & $42 \cdot 8$ & 30.0 & $30 \cdot 0$ & 44.9 & $67 \cdot 0$ & 30.0 & 30.0 & $67 \cdot 0$ & $56 \cdot 6$ & $30 \cdot 0$ & $30 \cdot 0$ & 58.4 & $75 \cdot 8$ & 30.0 & 30.0 & 62.3 \\
\hline
\end{tabular}

$\mathrm{R}$, reference scenario; 1 , best scenario; 2, moderate scenario; 3 , constant scenario. 
particular, the growth rates in younger age groups were higher (47.1\% in 1990 and $81.8 \%$ in 2016) compared with those in the rest of the population (40.9\% (50-69 years old) and $47.3 \%$ ( $\geq 70$ years old) in 1990 and $58.0 \%$ (50-69 years old) and $44.9 \%$ ( $\geq 70$ years old) in 2016). The Pearson's correlations between the DALY rates and fruit intake from 1990 to 2016 were statistically significant $(P<0.05$ for all combination of the diseases and sex-specific categorisation), except for the correlation among males who had neoplasms $(P=0 \cdot 216)$.

\section{Future trends of disability-adjusted life years rate for neoplasms, cardiovascular diseases and diabetes and kidney diseases}

\section{Neoplasms}

The estimated parameters $(p, d, q)$ in each autoregressive integrated moving average model defined by equation (2) and their Akaike Information Criteria are provided in online Supplemental Table 1. Figure 1 shows the trend of DALY rates for neoplasms for all-ages group by sex and scenario. Detailed and age group-specific DALY rates are provided in online Supplemental Figures 1-3 (1: 2049 years old, 2: 50-69 years old and 3: $\geq 70$ years old, respectively). Online Supplemental Figures 1-3 show that the DALY rates have decreasing trends through 2040 in every age- and sex-group (except for men aged $\geq 70$ years old) while the total population estimate shows an increasing trend. This discrepancy between the age group specific and total population estimates suggests that the latter was greatly affected by population ageing. In the reference scenario, the greatest decline in DALY rates was expected in the 50-69-year-old age group, with declines of 14.9, 8.9 and $13.6 \%$ for men, women and sex-combined groups, respectively, from 2016 to 2040. Exact predicted values of DALY rates in 2040 for 20-49 years old, 50-69 years old, $\geq 70$ years old and all-ages group and for men, women and both sex-combined groups and for all scenarios are presented in online Supplemental Table 2.

\section{Cardiovascular diseases}

Figure 2 shows the trends of DALY rates for cardiovascular diseases for all-ages group by sex and scenarios. More detailed and age group-specific DALY rates are provided in online Supplemental Figures 4-6 (4: 20-49 years old, 5: 50-69 years old and 6: $\geq 70$ years old, respectively). The DALY rates continued to decline through 2040 regardless of the scenarios, sex- and age-groups. In the reference scenario, the greatest decline in DALY rates was expected in the $\geq 70$-year-old age group, with average declines of $41.7,42.3$ and $39.3 \%$ for male, female and sex-combined groups from 2016 to 2040, respectively. Additionally, the DALY rates in sex- and all-ages groups show remarkable differences between the scenarios with nonoverlapping $95 \%$ prediction intervals (PI): DALY rates (95\% PIs) in 2040 were $4027.7(3857.5,4205.3)$ and $3837.9(3675.7$,
4007.1) for reference and constant scenarios, respectively, while DALY rates (95\% PIs) in 2040 were $3417 \cdot 3$ (3272.9, 3568.0) for better and moderate scenarios. Similar results were observed by age groups, particularly in men and sex-combined groups aged 20-49 years.

\section{Diabetes and kidney diseases}

Figure 3 shows the trends of DALY rates for diabetes and kidney diseases for all-ages group by sex and scenarios. More detailed and age group-specific DALY rates are provided in online Supplemental Figures 7-9 (7: 20-49 years old, 8: 50-69 years old and 9: $\geq 70$ years old, respectively). As with neoplasms' trend, the age group-specific DALY rates show a decreasing trend, while the DALY rates in all-ages group show an increasing trend, also suggesting that the effects of the population ageing on future DALY rate of diabetes and kidney diseases might be significant. Except for males aged 50-69 years, there was no remarkable difference in DALY rates between the scenarios with overlapping PI. In the reference scenario, the greatest decline in DALY rate was expected in the $\geq 70$-year-old age group, with average declines of $13.4,22 \cdot 1$ and $20.5 \%$ for male, female and sex-combined groups from 2016 to 2040.

\section{Discussion}

To optimize long-term medical investment and policy implementation, it is important to understand the future trajectories of health and drivers of health at the population level. In the current study, we predicted a set of cause-, sex- and age group-specific DALY rates for chronic diseases that have previously been associated with low fruit intake by assessing four future scenarios of the proportion of the Japanese population that consume $<100 \mathrm{~g}$ of fruit/d. Our analyses indicate that increasing fruit intake may have a potential to reduce disease burden and thereby contribute to addressing the major policy issue of increasing healthcare costs due to the ageing society and the extension of life expectancy ${ }^{(11)}$.

Overall, the DALY rates in the reference scenario were predicted to increase for neoplasms and decrease for cardiovascular diseases and diabetes and kidney diseases through 2040 (except for the sex-combined group for cardiovascular diseases, which showed the stable trend). Similarly, the age group-specific DALY rates for these three causes were predicted to decrease (except for male aged >70-year-old group, which shows a stable trend). Importantly, in all sex- and age-groups and among men aged 20-49 years, the predicted DALY rates attributable to cardiovascular diseases between four assumed scenarios did not overlap with one another greatly, suggesting that there is a significant potential for reducing the burden of cardiovascular diseases by increasing fruit intake at the population level. Our findings are in accordance with previous studies in Japan providing a clear inverse association 

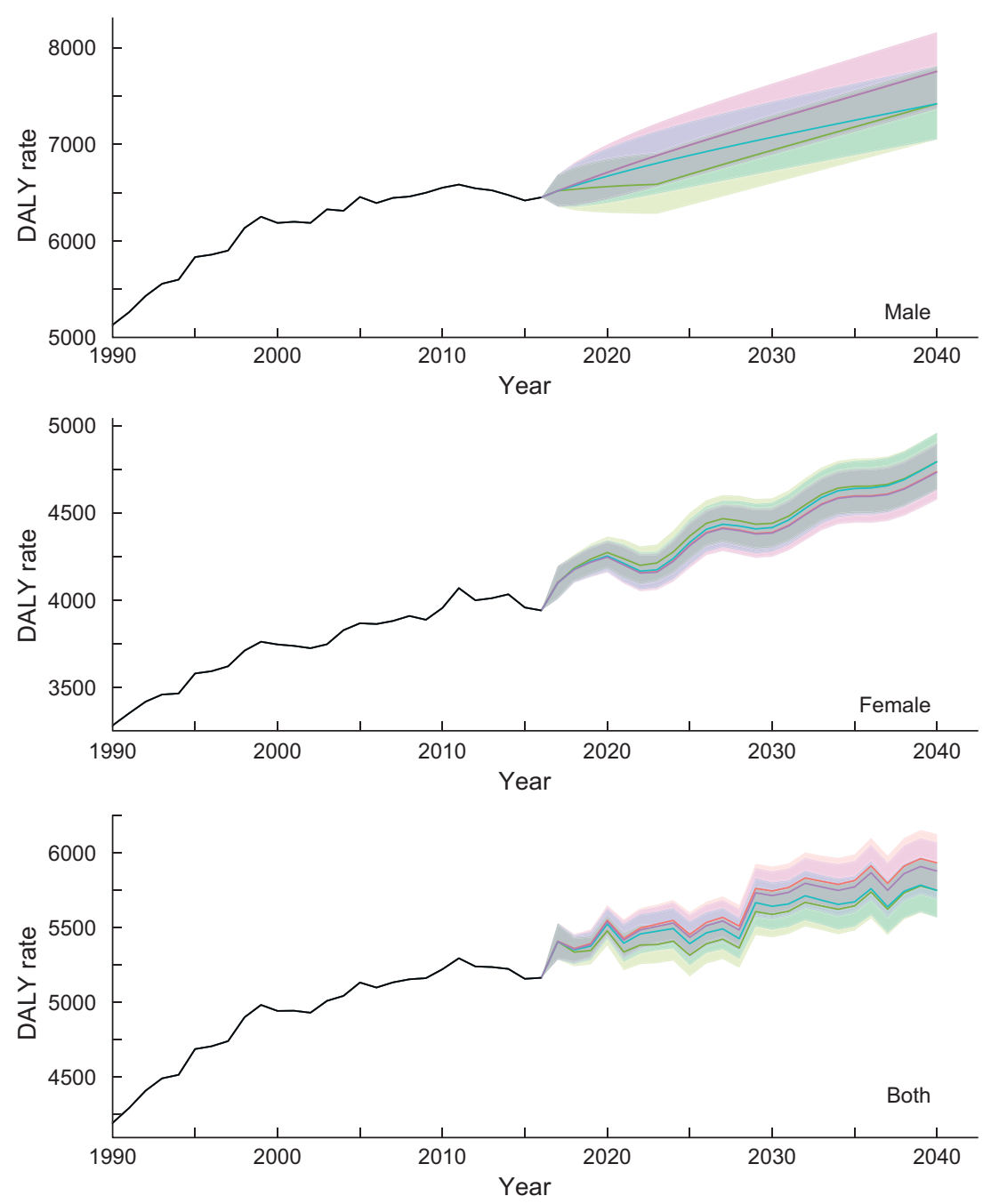

Fig. 1 (colour online) Observed and projected all ages disability-adjusted life years (DALY) rate (per 100 000) for neoplasms for reference forecast and three alternative scenarios, 1990-2040: male, female and both sexes combined. 1: best scenario, 2: moderate scenario, 3: constant scenario. It is important to note that the $y$-axis scales are different for each panel in order to make the differences between scenarios easier to understand. Detailed values are shown in online Supplemental Table 3. - , Observed; -, Reference; -, Scenario $1 ;=$, Scenario 2 ; -, Scenario 3

of fruit intake and risk of cardiovascular diseases and diabetes and kidney diseases. For example, Takachi et al. ${ }^{(20)}$, who conducted a survival analysis of participants aged 45-75 years old in several cohorts in Japan from 1990 to 2003, showed an inverse relationship between fruit intake and risk of cancer and cardiovascular diseases. Kurotani et $a l^{(21)}$ used the same data set and conducted a logistic regression analysis to indicate the association between high fruit intake and low risk of diabetes. Nagura et al. (2009) conducted a survival analysis with a different multi-cohort data set of participants aged 45-79 years from 1988 to $2003^{(22)}$ to reach the same conclusion for mortality risk on cardiovascular diseases.

Fruits are rich sources of potassium, folate, fibre and antioxidants, including vitamin $\mathrm{C}, \beta$-carotene and flavonoids ${ }^{(23)}$. Previous randomised controlled trials showed that increasing fruit (and vegetable) intake can reduce blood pressure as well as increase urinary K excretion ${ }^{(24)}$. Since raised blood pressure is a major risk factor for cardiovascular diseases, the effect of fruit to lower blood pressure could be one of the major biological mechanisms that leads to a reduced risk of cardiovascular diseases ${ }^{(25)}$. Limited evidence provides that as part of a healthy diet low in fat, sugars and salt/Na; fruits (and vegetables) may prevent weight gain and reduce the risk of obesity, a risk factor for the three diseases of our interest ${ }^{(21,26)}$. Additionally, the previous studies suggest the potential impact of fruit intake on shifting towards healthier and more sustainable diets globally ${ }^{(27,28)}$ as well as the effect for reducing the risk of cardiovascular diseases ${ }^{(20,22,27,28)}$. Our models do not support the notion that a substantial benefit from increased fruit intake can be obtained with respect to neoplasms and diabetes and kidney diseases (i.e. prediction trajectories among most scenarios have 
Predication of disability-adjusted life years due to low fruit intake


Fig. 2 (colour online) Observed and projected all ages disability-adjusted life years (DALY) rate (per 100 000) for cardiovascular diseases for reference forecast and three alternative scenarios, 1990-2040: male, female and both sexes combined. 1: best scenario, 2: moderate scenario, 3: constant scenario. It is important to note that the $y$-axis scales are different for each panel in order to make the differences between scenarios easier to understand. Detailed values are shown in Supplemental Table 4. - - Observed; -, Reference; =, Scenario 1; =, Scenario 2; =, Scenario 3

overlapping prediction intervals). It suggests that there may be other risk factors at play, including physical activity and social determinants of health such as physical environment and social relationships with other persons, all of which have more significant impact on the three disease groups of interest ${ }^{(2,29-31)}$. It is our ongoing study to merge our data set with other data sources that include possible confounders to adjust for the statistical association between the DALY rates and fruit intake.

The ten-year health promotion guideline 'Health Japan 21 (second phase)' has focused on nation-wide campaigns to raise awareness and to induce behavioural change for increasing fruit intake. At the same time it has also tried to encourage the food industry and the Japanese society at large to promote the consumption of a balanced diet, including the adequate amount of fruit intake. As we have shown, the proportion of those who consume $<100 \mathrm{~g}$ of fruit/d in Japan has been increasing since 1990 and reached $62 \cdot 3 \%$ in $2016^{(4,5,10)}$. This is far from the target of Health Japan 21. WHO recommends several effective actions for policy-makers to create a healthy food environment; for example, by applying school interventions that encourage children to adopt and maintain a healthy diet and exploring economic incentives or disincentives, including taxation and subsidies, to promote a healthy diet ${ }^{(8,32,33)}$. Since low fruit intake is a modifiable factor application of the interventions presented in WHO's guidelines, clinical and political actions are urgently demanded to prevent disease, prolong healthy life and efficiently maintain and improve population health ${ }^{(11)}$.

The strengths of our study lie in the fact that we use the best available data that represent the Japanese 

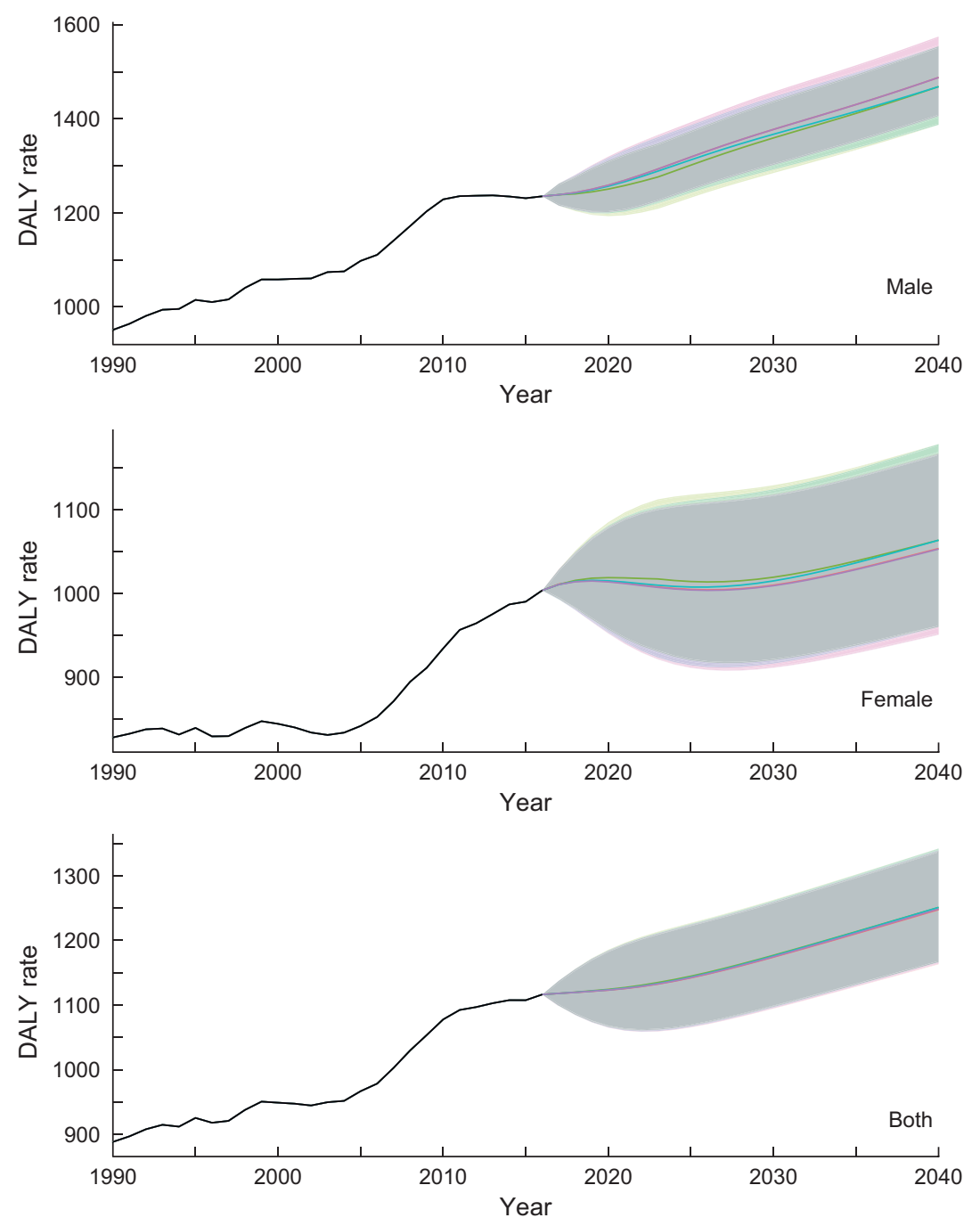

Fig. 3 (colour online) Observed and projected all ages disability-adjusted life years (DALY) rate (per 100 000) for diabetes and kidney disease for reference forecast and three alternative scenarios, 1990-2040: male, female and both sexes combined. 1: best scenario, 2: moderate scenario, 3: constant scenario. It is important to note that the $y$-axis scales are different for each panel in order to make the differences between scenarios easier to understand. Detailed values are shown in online Supplemental Table 5. - , Observed; -, Reference; -, Scenario 1; =, Scenario 2; -, Scenario 3

population's dietary pattern over time with a flexible time series modelling approach. Simple models as ours have advantages in allowing for a prompt exploration of dietary risk factors and relevant disease burden forecasts. Our study, however, has limitations. First, several risk predictors, which were not included in our models, could change the prediction results. For example, blood pressure, which is known to be associated with cardiovascular diseases and diabetes and kidney diseases ${ }^{(34-36)}$, was not included in our models because of data availability. We welcome re-evaluation of our results by using more detailed data of risk predictors. Second, in Japan, health outcomes are largely explained by health system performance ${ }^{(11)}$. The prediction may be influenced by these health drivers more than by individual risk factors. Third, while DALY is a well-established population health measure that is widely used as an important benchmark for making health policy decision, it has many limitations as discussed elsewhere ${ }^{(37)}$. In particular, DALY cannot clearly answer the question about how much investment is required to reduce the burden of disease. Finally, our study is also subject to similar limitations as other studies concerning dietary patterns ${ }^{(38,39)}$. In the National Health and Nutrition Survey, dietary intake was estimated based on self-report and recorded in a single day, which might not represent a long-term and/or seasonal dietary pattern. Additionally, data based on self-reporting may be subject to bias, such as the social-desirability-response bias ${ }^{(17,40)}$ and measurement error in comparison to medical records or laboratory-confirmed data.

In conclusion, our prediction results show that higher fruit intake is associated with lower disease burden in 
Japan. Further research is required to assess which policies and interventions can be used to achieve an increase in fruit intake as modelled in the scenarios of the current study. In addition, our analytical framework using autoregressive integrated moving average model might be useful for other countries by providing information for assessing how the control of fruit intake can impact the population health.

\section{Acknowledgements}

Acknowledgement: None. Financial support: This manuscript is an original work that has not been submitted for consideration or published elsewhere. The current study is partially supported by a joint research grant from Ajinomoto Co. Inc., and Grants-in-Aid for Scientific Research from the Ministry of Education, Culture, Sports, Science and Technology of Japan (MEXT). K.N., H.U. and N.H. are employed by the commercial founder, Ajinomoto Co. Inc. The commercial funder provided support in the form of salaries for K.N., H.U. and N.H., but did not have any additional role in the study design, data collection and analysis, decision to publish or preparation of the manuscript. The specific roles of these authors are articulated in the author contributions' section. The other funder (MEXT) had also no role in the study design, data collection and analysis, decision to publish or preparation of the manuscript. Conflict of interest: S.N. and K.S. report grants from Ajinomoto Co. Inc. and the Ministry of Education, Culture, Sports, Science and Technology of Japan. D.Y., S.N., S.T., A.I., U.P., S.K.R. and K.S declare no competing interests. K.N., H.U. and N.H. declare that they are employed by Ajinomoto Co. Inc. and have no other competing interests. Authorship: S.N. led the study. D.Y. and S.N. conceived and designed the study. All authors took responsibility for the integrity of the data and the accuracy of the data analysis. D.Y., S.N., S.T. and K.S. acquired the data. D.Y., S.N., S.T., P.U., S.K.R., K.N., H.U. and N.H. analysed and interpreted the data. D.Y. conducted the statistical analysis. D.Y. and S.N. drafted the article. All the authors made critical revisions to the manuscript for important intellectual content and gave final approval of the manuscript. The opinions, results and conclusions reported in this paper are those of the authors and are independent from the funding bodies. Ethics of buman subject participation: Ethical approval for the study was granted by the ethics committee of The University of Tokyo (11964). Written informed consent was not required, as this study was a secondary analysis of anonymised data that is collected routinely by the MHLW. Data of GBD 2017 are also secondary, aggregated estimates by country, sex and age groups.

\section{Supplementary material}

For supplementary material accompanying this paper visit https://doi.org/10.1017/S1368980020004541

\section{References}

1. GBD 2017 Risk Factor Collaborators (2018) Global, regional, and national comparative risk assessment of 84 behavioural, environmental and occupational, and metabolic risks or clusters of risks for 195 countries and territories, 1990-2017: a systematic analysis for the Global Burden of Disease Study 2017. Lancet 392, 1923-1994.

2. GBD 2017 Diet Collaborators (2019) Health effects of dietary risks in 195 countries, 1990-2017: a systematic analysis for the Global Burden of Disease Study 2017. Lancet 393, 1958-1972.

3. Food and Agriculture Organization of the United Nations (2013) FAOSTAT Statistical Database. http://www.fao.org/ faostat/en/?\#home (accessed October 2019).

4. Katanoda K \& Matsumura Y (2002) National Nutrition Survey in Japan. Its methodological transition and current findings. J Nutr Sci Vitaminol 48, 423-432.

5. Ministry of Health Labour and Welfare (2017) The National Health and Nutrition Survey in Japan, 2016. Tokyo: Ministry of Health, Labour and Welfare.

6. Mo X, Gai RT, Sawada K et al. (2019) Coronary heart disease and stroke disease burden attributable to fruit and vegetable intake in Japan: projected DALYS to 2060. BMC Public Health 19, 707.

7. Ministry of Health Labour and Welfare (2012) A Basic Direction for Comprehensive Implementation of National Health Promotion. https://www.mhlw.go.jp/file/06-Seisaku jouhou-10900000-Kenkoukyoku/0000047330.pdf (accessed December 2019).

8. World Health Organization (2018) Healthy Diet. https:// www.who.int/news-room/fact-sheets/detail/healthy-diet (accessed April 2020).

9. Ministry of Health Labour and Welfare (2018) Social Security and Workplace Reform with a View to 2040. https://www. mhlw.go.jp/stf/shingi/other-syakaihosyou_306350_00001. html (accessed October 2019).

10. Ministry of Health Labour and Welfare (2018) Healthy Life Extension Plan. https://www.mhlw.go.jp/content/ 12601000/000514142.pdf (accessed December 2019).

11. Nomura S, Sakamoto H, Glenn S et al. (2017) Population health and regional variations of disease burden in Japan, 1990-2015: a systematic subnational analysis for the Global Burden of Disease Study 2015. Lancet 390, 1521-1538.

12. Foreman KJ, Marquez N, Dolgert A et al. (2018) Forecasting life expectancy, years of life lost, and all-cause and causespecific mortality for 250 causes of death: reference and alternative scenarios for 2016-40 for 195 countries and territories. Lancet 392, 2052-2090.

13. GBD 2017 DALYs \& HALE Collaborators (2018) Global, regional, and national disability-adjusted life-years (DALYs) for 359 diseases and injuries and healthy life expectancy (HALE) for 195 countries and territories, 1990-2017: a systematic analysis for the Global Burden of Disease Study 2017. Lancet 392, 1859-1922.

14. GBD 2017 Mortality Collaborators (2018) Global, regional, and national age-sex-specific mortality and life expectancy, 1950-2017: a systematic analysis for the Global Burden of Disease Study 2017. Lancet 392, 1684-1735. 
15. Kanazawa M, Yoshiike N, Osaka T et al. (2005) Criteria and classification of obesity in Japan and Asia-Oceania. World Rev Nutr Diet 94, 1-12.

16. Haneda M, Noda M, Origasa H et al. (2018) Japanese clinical practice guideline for diabetes 2016. Diabetol Int 9, 1-45.

17. Ikeda N, Takimoto H, Imai S et al. (2015) Data resource profile: the Japan National Health and Nutrition Survey (NHNS). Int J Epidemiol 44, 1842-1849.

18. Ministry of Education Culture Sports Science and Technology (2015) Standards tables of food composition in Japan-2015 (seventh revised edition). http://www. mext.go.jp/en/policy/science_technology/policy/title01/ detail01/sdetail01/sdetail01/1385122.htm (accessed December 2019).

19. Hamilton JD \& Princeton University P (1994) Time Series Analysis. New Jersey: Princeton University Press.

20. Takachi R, Inoue M, Ishihara J et al. (2008) Fruit and vegetable intake and risk of total cancer and cardiovascular disease: Japan Public Health Center-Based Prospective Study. Am J Epidemiol 167, 59-70.

21. Kurotani K, Nanri A, Goto A et al. (2013) Vegetable and fruit intake and risk of type 2 diabetes: Japan Public Health Center-based prospective study. Br J Nutr 109, 709-717.

22. Nagura J, Iso H, Watanabe Y et al. (2009) Fruit, vegetable and bean intake and mortality from cardiovascular disease among Japanese men and women: the JACC Study. $\mathrm{Br} J$ Nutr 102, 285-292.

23. Liu RH (2013) Health-promoting components of fruits and vegetables in the diet. Adv Nutr $\mathbf{4}, 384$ S-392S.

24. Appel LJ, Moore TJ, Obarzanek E et al. (1997) A clinical trial of the effects of dietary patterns on blood pressure. DASH Collaborative Research Group. $N$ Engl J Med 336, $1117-1124$.

25. He FJ, Nowson CA \& MacGregor GA (2006) Fruit and vegetable consumption and stroke: meta-analysis of cohort studies. Lancet 367, 320-326.

26. Ledoux TA, Hingle MD \& Baranowski T (2011) Relationship of fruit and vegetable intake with adiposity: a systematic review. Obes Rev 12, e143-e150.

27. Wang X, Ouyang Y, Liu J et al. (2014) Fruit and vegetable consumption and mortality from all causes, cardiovascular disease, and cancer: systematic review and dose-response meta-analysis of prospective cohort studies. BMJ 349, g4490.

28. Mason-D'Croz D, Bogard JR, Sulser TB et al. (2019) Gaps between fruit and vegetable production, demand, and recommended consumption at global and national levels: an integrated modelling study. Lancet Planet Health 3, e318-e329.

29. Schwingshackl L, Knuppel S, Michels N et al. (2019) Intake of 12 food groups and disability-adjusted life years from coronary heart disease, stroke, type 2 diabetes, and colorectal cancer in 16 European countries. Eur J Epidemiol 34, 765-775.

30. World Cancer Research Fund (2018) Diet, Nutrition, Physical Activity and Cancer: A Global Perspective-Continuous Update Project Expert Report. London: WCRF International.

31. Braveman P \& Gottlieb L (2014) The social determinants of health: it's time to consider the causes of the causes. Public Health Rep 129, 19-31.

32. World Health Organization (2013) Global Action Plan for the Prevention and Control of Noncommunicable Diseases 2013-2020. Geneva: World Health Organization.

33. World Health Organization (2006) Global strategy on diet, physical activity and health: a framework to monitor and evaluate implementation.

34. Brunstrom M \& Carlberg B (2018) Association of blood pressure lowering with mortality and cardiovascular disease across blood pressure levels: a systematic review and meta-analysis. JAMA Intern Med 178, 28-36.

35. Jafar TH, Stark PC, Schmid CH et al. (2003) Progression of chronic kidney disease: the role of blood pressure control, proteinuria, and angiotensin-converting enzyme inhibition: a patient-level meta-analysis. Ann Intern Med 139, 244-252.

36. Lv J, Ehteshami P, Sarnak MJ et al. (2013) Effects of intensive blood pressure lowering on the progression of chronic kidney disease: a systematic review and meta-analysis. CMAJ 185, 949-957.

37. Anand S \& Hanson K (1997) Disability-adjusted life years: a critical review. J Health Econ 16, 685-702.

38. Kurotani K, Akter S, Kashino I et al. (2016) Quality of diet and mortality among Japanese men and women: Japan Public Health Center based prospective study. BMJ 352, i1209.

39. Oba S, Nagata C, Nakamura K et al. (2009) Diet based on the Japanese Food Guide Spinning Top and subsequent mortality among men and women in a general Japanese population. J Am Diet Assoc 109, 1540-1547.

40. Subar AF, Freedman LS, Tooze JA et al. (2015) Addressing current criticism regarding the value of self-report dietary data. J Nutr 145, 2639-2645. 